\title{
Tuberculin Skin Test (TST) Indurations in Smear Positive TB Patients and Healthy Individuals in Calabar, Nigeria
}

\author{
Anne E. Asuquo, ${ }_{1}^{1}$ Adebayo N. Dairo, ${ }^{1}$ Lydia Abia-Bassey, ${ }^{1}$ Martin M. Meremiku ${ }^{2}$ and \\ Benjamin P. Thumamo ${ }^{1}$ \\ ${ }^{1}$ Department of Medical Microbiology and Parasitology, ${ }^{2}$ Department of Paediatrics, College of \\ Medical Sciences, University of Calabar, Calabar, Nigeria
}

KEYWORDS Tuberculin Skin Test. Tuberculosis. Control. Nigeria

\begin{abstract}
The tuberculin skin test (TST) is used to detect latent Mycobacterium tuberculosis infection. WHO guidelines recommend $10 \mathrm{~mm}$ as tuberculosis (TB) infection threshold for high risk individuals and $15 \mathrm{~mm}$ for persons with no risk factors for TB. Nigeria is one of the 22 high burden countries for TB and her population is at risk of exposure to the TB germ. In an attempt to investigate indigenous TST indurations in a local population, 200 apparently healthy new students of tertiary institutions and other residents of Calabar - Nigeria metropolis that required the test for routine medical examination were examined. Each subject was injected intradermally with $0.1 \mathrm{~mL}$ of 5TU of purified protein derivative (PPD) into the dorsal surface of the forearm. Indurated areas were measured after 48-72 hours of administration and results expressed in millimeters. 200 smear positive TB patients receiving treatment at the treatment center of the National TB Control program were used as the reference group. Results were statistically analysed using Chi square and T-test. Data obtained from the apparently healthy group show that approximately $31 \%$ of the individuals had indurations measuring $5-7 \mathrm{~mm}$ while $29 \%$ were non-reactive. At least $25 \%$ of these individuals had indurations of $>10 \mathrm{~mm}$ compared to $95 \%$ of TB patients. Nine $(4.5 \%)$ of TB patients have indurations $<10 \mathrm{~mm}$. The results of this study imply that at least $25 \%$ of the healthy subjects are at risk of progressing to active disease when exposed to conditions that lower the individual's immune status.
\end{abstract}

\section{INTRODUCTION}

Tuberculin skin testing (TST) is a delayed type hypersensitivity reaction in form of induration seen at the test site of an individual sensitized to the tuberculin antigen. It is the principal means of detecting latent $M$. tuberculosis infection. TST is also used widely to confirm clinical suspicion of TB especially in children living in less developed countries, where laboratories are ill equipped. It has been used to estimate the burden of the disease in epidemiological studies (Snider 1982). Screening for tuberculosis infection is recommended for asymptomatic high risk persons including persons infected with HIV, contacts of persons with active TB, immigrants from high TB burden countries, persons of low socioeconomic status among others (CDC 1994; Green 1994). In some populations, screening for TB infection is required by law (CDC 1993). A high percentage of people living in developing countries like Nigeria also live in conditions of social deprivation, with no access to adequate medical care.

Correspondence to:

Benjamin Thumamo

E-mail: thumamo@yahoo.fr
The rationale for detection of tuberculosis (TB) infection in these groups is to provide clinical intervention in individuals that meet set criteria in order to prevent the development of TB disease (ATS 1994). On the basis of observational data and expert opinions in the USA, minimal criteria for defining TB infection have been set for various groups of persons: $15 \mathrm{~mm}$ diameter for low-risk individuals, $10 \mathrm{~mm}$ for high risk individuals (immigrants, persons of low socio-economic status, people with conditions that increase TB risk, etc) and $5 \mathrm{~mm}$ for persons at very high-risk (HIV infected persons, contacts of TB patients, etc) (CDC 1995). Our study of tuberculin reactions in an indigenous population was an attempt to provide data on baseline indurations in the population. Currently, no data exist on reactor rates for our population.

\section{MATERIALS AND METHODS}

Study Population: Subjects for this study were drawn from a healthy population and comprised new entrants into tertiary institutions that were undergoing routine medical examination as part of their admission requirements as well as other residents of Calabar that visited the Infectious Disease Hospital (IDH) for routine 
medical examinations. The students were from various parts of the country where they resided until their recent residence in Calabar. They represent an Eastern Nigeria population. The control group (TB patients) comprised persons resident in Calabar that were receiving treatment from the National TB Control Programme treatment centre.

Sample Size: Four hundred subjects were studied: 200 apparently healthy individuals and 200 Smear positive TB patients.

Demographic Data: Information on the demography and other relevant data required for the study were collected through the administration of questionnaires.

Mantoux Test: In the healthy population, this test was performed using 5TU of the purified protein derivative (PPD) (BB-NCIPD Ltd, Sofia, Bulgaria). With the aid of a disposable tuberculin syringe, a volume of $0.1 \mathrm{~mL}$ of the antigen was injected intra-dermally into the dorsal surface of the forearm of each individual. The test was read after 48-72 hours of administration and the size of the indurated area was recorded in millimeters. For the control group, the TST indurations of the individuals were obtained from medical records of the patients at the IDH (Infectious Diseases Hospital), Calabar.

Analysis of Results: Results were statistically analysed using Chi-square and t-test.

\section{RESULTS}

The readings obtained from indurated areas following the administration of 5TU of purified protein derivative (PPD) on healthy individuals and TB patients are shown in Figure 1. The frequency of occurrence of tuberculin sizes shows that $29 \%$ of apparently healthy individuals were non-reactive to the tuberculin antigen. The most frequent tuberculin size range among apparently healthy individual was $5-7 \mathrm{~mm}(31 \%)$ while that in TB patients were $10-12 \mathrm{~mm}(36 \%)$ and $13-15 \mathrm{~mm}(31 \%)$. Tuberculin sizes in about $18 \%$ of apparently healthy individuals ranged from $10-12 \mathrm{~mm}$. The induration area of $10 \mathrm{~mm}$ provided by the $\mathrm{CDC}$ as diagnostic of TB infection in high risk groups was used as threshold in the tested population. Table 1 shows that $75 \%$ of the apparently healthy individuals had indurations below $10 \mathrm{~mm}$ compared to $4 \%$ of TB patients.

Table 1: Association between TB patients and apparently healthy individuals induration sizes to tuberculin skin testing.

\begin{tabular}{|c|c|c|c|}
\hline Induration size & $<10 \mathrm{~mm}$ & $>=10 \mathrm{~mm}$ & Total \\
\hline TB patients & $8(4 \%)$ & $192(96 \%)$ & 200 \\
\hline $\begin{array}{l}\text { Apparently } \\
\text { healthy individua }\end{array}$ & $\begin{array}{l}150(75 \%) \\
\text { als }\end{array}$ & $50(25 \%)$ & 200 \\
\hline Total & $158(39.5 \%)$ & $242(60.5 \%)$ & 400 \\
\hline
\end{tabular}

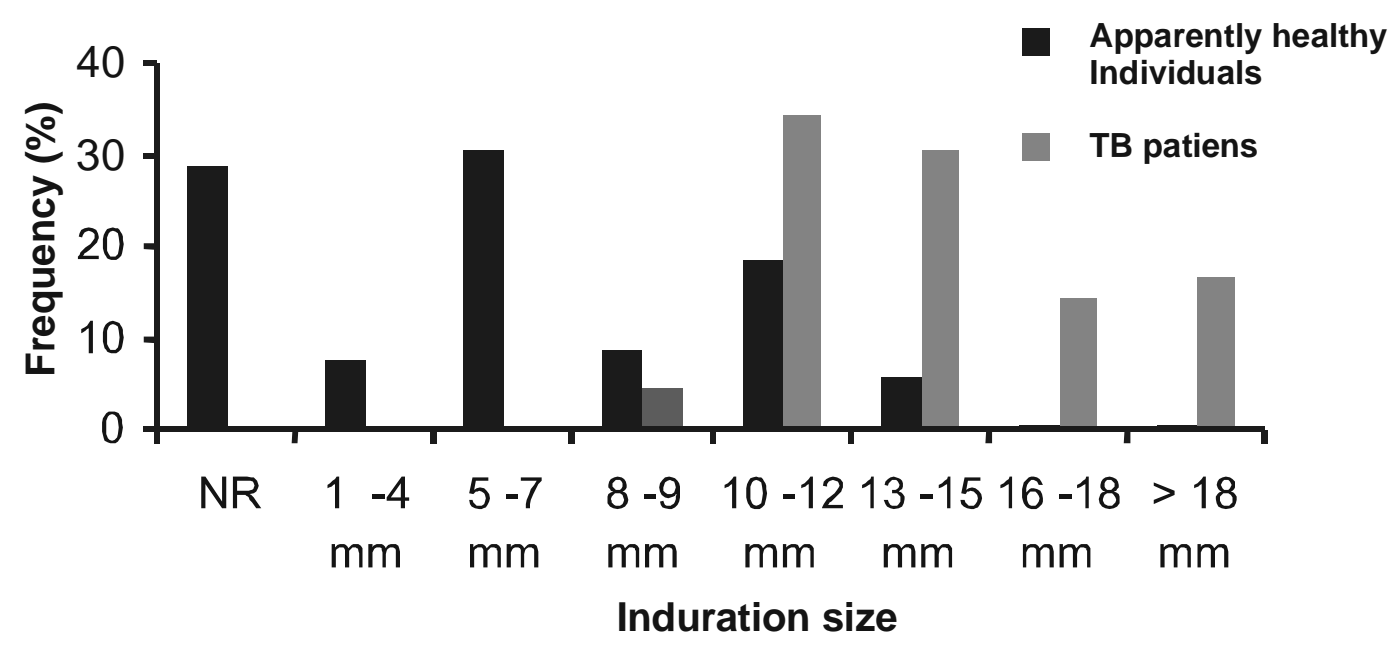

Fig. 1. Frequency distribution of tuberculin size among TB patients and apparently healthy individuals in Calabar

NR = Non Reactive 
Indurations of $10 \mathrm{~mm}$ or more were found in $96 \%$ of TB patients and $25 \%$ of healthy individuals. The tuberculin sizes obtained from healthy individuals and TB patients were plotted on a scatter graph (Fig. 2) using $10 \mathrm{~mm}$ as threshold for infection. The mean value for indurations for TB patients was $14.5 \mathrm{~mm}$ while that of healthy group was $5.5 \mathrm{~mm}$. Indurations for $\mathrm{TB}$ patients were significantly higher than indurations for healthy subjects $(\mathrm{P}<0.05)$. Two apparently healthy individuals in this study have tuberculin sizes of $>15 \mathrm{~mm}$.

Irrespective of gender, the mean of the areas of induration among healthy individuals was 5.5 $\mathrm{mm}$ while that of patients was $14.5 \mathrm{~mm}$. There was no statistical significance $(\mathrm{P}>0.05)$ between gender and size of area of induration in both TB patients and healthy individuals. However, $4.6 \%$ of the 108 males compared to $3.3 \%$ of the 92 female TB patients had indurations of less than $10 \mathrm{~mm}$. Similarly, indurations of $>=10$ were observed in $95.4 \%$ males while $96.7 \%$ females had indurations above $10 \mathrm{~mm}\left(\chi^{2}=0.24\right)$. Among the healthy individuals, $74 \%$ of males and $76 \%$ of females had indurations of $<10 \mathrm{~mm}$ while $26 \%$ and $24 \%$ respectively of males and females had indurations of $>=10 \mathrm{~mm} .\left(\chi^{2}=0.107\right)$.
In this study, there was a strong correlation between age and the distribution of TST indurations among TB patients and the apparently healthy individuals $(\mathrm{P}<0.05)$. Among TB patients, $29(14.5 \%)$ were aged $=<15$ years, $74(37 \%)$ between 16 and 30 years, and 97 (48.5\%) above 30 years, and of the 200 apparently healthy individuals, $27(13.5 \%)$ were aged $=<15,139$ $(69.5 \%)$ between 16 and 30 years, and 34 (17\%) above 30 years $\left(C^{2}=50.2\right)$ (Table 2$)$.

Table 2: Age distribution of apparently healthy individuals and TB patients

\begin{tabular}{lrcrr}
\hline Age (years) & $=<15$ & $>15-=<30$ & $>30$ & Total \\
\hline TB patients & 29 & 74 & 97 & 200 \\
$\begin{array}{l}\text { Apparently } \\
\text { healthy }\end{array}$ & 27 & 139 & 34 & 200 \\
$\quad$ individuals & & & & \\
\hline Total & 56 & 213 & 131 & 400 \\
\hline
\end{tabular}

\section{DISCUSSION}

A positive TST is the only evidence of latent tuberculosis infection and the larger the tuberculin reactivity, the higher the risk of progression to active tuberculosis (Kheder et al. 2000). In this survey of tuberculin reactions

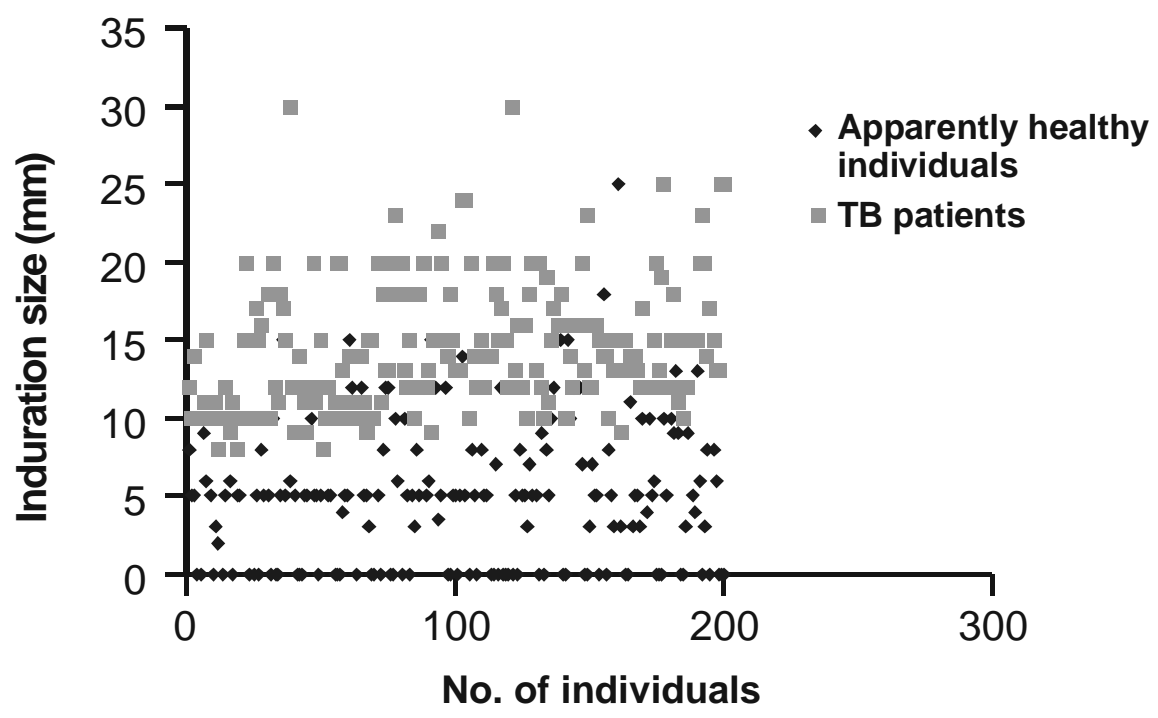

Fig. 2. Scatter graph showing threshold of induration size at $10 \mathrm{~mm}$ among TB patients and apparently healthy individuals in Calabar 
among a healthy Nigerian population, we compared TST reactor rates in an indigenous Nigerian population to those found in smear positive TB patients. There is no known study providing such data currently. Data obtained from this study are expected to provide some insight into the reservoir of reactivation TB in the healthy population.

Taking into account the mean of both apparently healthy individuals and TB patients ( $5.5 \mathrm{~mm}$ and $14.5 \mathrm{~mm}$ respectively) in this study, the $10 \mathrm{~mm}$ indurations of TST prescribed for TB infection in high risk groups remains a strong indicator $(p<0.0005)$ of TB infection/disease in Calabar. The percentage of tuberculin reactors with indurations of $>10 \mathrm{~mm}(25 \%)$ among our healthy subjects demonstrate that there is a milieu for reactivation tuberculosis in this population particularly in the presence of factors such as HIV infection, poverty, malnutrition, poor housing conditions among others. According to Brooks et al. (2004), 90\% of persons with $10 \mathrm{~mm}$ indurations have $M$. tuberculosis infection and the lifetime risk of reactivation tuberculosis is $20 \%$ or more among such persons.

Evidence abound that there is increased reactivation of latent tuberculosis and increased transmission of it due to the immunodeficiency associated with HIV disease (Katz 1997). However, whether or not a particularly infected person will become ill is impossible to predict with certainty. Nonetheless, it has been estimated that $5 \%$ of infected persons take ill within $12-24$ months of being infected; another $5 \%$ heal initially but, after years or decades, develop active tuberculosis either in the lungs or elsewhere in the body.

The HIV epidemic has increased the need for detecting and treating latent $M$. tuberculosis infection. Unfortunately, despite the fact that the best public health strategy is to prevent disease before it develops, for reasons bordering on cost, latent TB is not treated in the poorer developing countries. In this study, a significant proportion (39\%) of apparently healthy individuals had indurations between 5 and $10 \mathrm{~mm}$. Nigeria is endemic for tuberculosis and most individuals may have been exposed to the TB germ during the course of their lives. Cross-reacting atypical Mycobacteria as well as Bacilli Calmette-Guerin (BCG) vaccination can also produce intermediate size reactions (ATS 1990; Mellor 1985). However, $\mathrm{BCG}$ vaccination is unlikely to produce the size of indurations in this study as most of our subjects in this study were adults and may have lost the immunity acquired from BCG (Skotniski 1993; Snider Jr. 1985). Without compromised immune status, the threat of progression to active disease in these individuals may be minimal. Of the $36 \%$ apparently healthy individuals that had $<5 \mathrm{~mm}$ indurations, $29 \%$ were non-reactive. While this may indicate lower likelihood of progression to active or inactive disease (CDC 1995), anergy to PPD is a common feature among HIV infected individuals and this does not preclude to developing the disease (Toivgoogiin et al., 2005). Although retesting is sometimes recommended for negative results, this study did not follow up TST negative individuals to skin test for anergy. Detection of latent TB is essential for HIV infected persons because the rate of reactivation for such individuals has been put at $10 \%$ annually compared to lifetime risk of 5-10\% in HIV seronegative individuals.

This study showed no relationship between gender and area of induration among apparently healthy individuals or TB patients This may imply that while the incidence of TB may be higher among male than female (Lienhardt et al. 2003; Lienhardt et al. 2005), immune reactivity which is a physiological process may not be gender dependent. In a study conducted among attendees at a methadone clinic, MacGregor et al. (1994) observed that gender was not strongly correlated with tuberculin reaction sizes. This study demonstrated a correlation between age and size of induration, implying an annual increase in infection due to reservoir of $M$. tuberculosis or mycobacteria other than tuberculosis (MOTT) in the environment. According to Lienhardt et al. (2003), the risk of being tuberculin skin test positive increases with age, implying that the $27 \%$ of apparently healthy individuals above 15 years with TST $>=10 \mathrm{~mm}$ in this study are more likely to develop the disease with increased age. The ages when infection is more likely to yield active disease are infancy, aged 15 - 25 and the elderly year (Brooks et al. 2004). The 16 apparently healthy children aged $>=5$ years in this study where contacts of TB patients or children being investigated following clinical suspicion of TB. Eleven of them were none reactive to the tuberculin antigen, however induration of 10 to $12 \mathrm{~mm}$ were observed in two cases. Thirty-five of the forty one adult that were none reactive were aged between 16 and 30 years. The reason for this uncertain lack of encounter 
with the TB germ though possible is unlikely in an endemic country like Nigeria. Anergy to the TB germ is a frequent feature of HIV infected persons. HIV status of individuals was not investigated.

\section{CONCLUSION}

The apparently healthy individuals of this study though not contagious, do form a pool of infected patients who may progress to active disease at a later date. The prevention of active tuberculosis through the treatment of latent tuberculosis infection could be a major element of the national strategy for eliminating tuberculosis. Targeted treatment for persons who are at the highest risk for reactivation tuberculosis will be needed to achieve this goal (Horsburgh 2004). Therefore, there is need for increased facilities for diagnosis, and targeted screening and treatment of this population. In this study, the group at the highest risk of reactivation $\mathrm{TB}$ comprises the $1 \%$ of healthy individuals whose indurations are $\geq 15 \mathrm{~mm}$, similar to the mean indurations for $\mathrm{TB}$ patients.

\section{ACKNOWLEDGEMENT}

We are grateful to the management and staff of the Infectious Diseases Hospital-Calabar, for providing technical support and TST data on the TB patients used in this study.

\section{REFERENCES}

ATS 1990. Diagnostic standards and classification of tuberculosis. Am Rev Respir Dis, 142: 725-735.

ATS 1994. Treatment of tuberculosis and tuberculosis infection in adults and children. Am J Respir Crit Care Med, 149: 1359-1374.

Brooks GF, Butel JS, Morse SA 2004. Cases and clinical correlations, In International Edition (23rd Ed.): Jatwetz, Melnick and Adelberg's Medical Microbiology. Singapore. McGraw-Hill Companies, pp. 734-70.
CDC 1993. Tuberculosis control laws-United States 1993. $M M W R, 42(\mathrm{RR}-15): 1-28$.

CDC 1994. Tuberculosis morbidity-United States 1993, MMWR, 43: 361-366.

CDC 1995. Tuberculosis morbidity-United States, 1994, MMWR, 44: 387-389, 395.

Green M 1994. Bright Futures: guidelines for Health Supervision of Infants, Children and Adolescents. Arlington, VA: National Center for Education in Maternal and Child Health.

Horsburgh CR 2004. Priorities for the treatment of latent tuberculosis infection in the United States. NEJM, 350: 2060-2067.

Katz DL 1997. Epidemiologic approaches, contributions and issues, In Epidemiology, Biostatistics and Preventive Medicine Review. Philadelphia, U.S.A. W.B. Saunders Company, pp. 3-8.

Kheder al Zahrani, Hamdan al Jahdali, Dick Menzies 2000. Does Size Matter? Utility of Size of Tuberculin Reactions for the Diagnosis of Mycobacterial Disease. Am J Respir Crit Care Med, 162: 1419-1422.

Lienhardt C, Fielding K, Sillah J, Tunkara A, Donkor S, Manneh K, Warndorff D, McAdam K, Bennett S 2003. Risk Factors for Tuberculosis Infection in Sub-Saharan Africa. A Contact Study in The Gambia. Am J Respir Crit Care Med, 168: 448-455.

Lienhardt C, Fielding K, Sillah JS, Bah B, Gustafson P, Warndorff D, Palayew M, Lisse I, Donkor S, Diallo S, Manneh K, Adegbola R, Aaby P, Bah-Sow O, Bennett S, McAdam K 2005. Investigation of the risk factors for tuberculosis: a case-control study in three countries in West Africa. Intern J Epidemiol, 34: 914-923.

MacGregor RR, Dunbar D, Graziani AL 1994. Tuberculin Reactions Among Attendees at a Methadone Clinic: Relation to Infection with the Human Immunodeficiency Virus. Clin Infect Dis, 19: 1100-4.

Mellor J 1985. False-positive results of Mantoux tests. Can Med Assoc J, 132: 1403.

Skotniski EM 1993. Post-BCG tuberculin testing: interpreting results and establishing essential baseline data. Can J Public Health, 84: 307-308.

Snider DE 1982. The tuberculin skin test. Am Rev Respir Dis, 125:108-118.

Snider DE Jr 1985. Bacille Calmette-Guerin vaccinations and tuberculin skin tests. JAMA, 253: 3438-3439.

Toivgoogiin A, Toyota M, Yasuda N, Ohara H 2005. Validity of using tuberculin skin test erythema measurement for contact investigation during a tuberculosis outbreak in school children previously vaccinated with BCG. J Epidemiol, 15:56-64. 